\title{
Die Gestaltung städtischer Abschließung im 21. Jahrhundert
}

\author{
L. Wacquant \\ Department of Sociology, 410 Barrows Hall, University of California, Berkeley, Berkeley, CA 94720, USA \\ Correspondence to: L. Wacquant (loic@ berkeley.edu)
}

Received: 2 August 2012 - Revised: 20 December 2012 - Accepted: 21 December 2012 - Published: 22 July 2014

\begin{abstract}
Kurzfassung. This paper draws on my books Urban Outcasts and Punishing the Poor, on the transformation of the forms and policy management of marginality in advanced society, to probe the use of space as a medium for social closure and control in the city. This first part sketches a framework for the (comparative) analysis of sociospatial seclusion, the process whereby particular social categories and activities are corralled and isolated in a reserved and restricted quadrant of physical and social space. The second part applies this schema to present a compressed analysis of the divergent trajectories of the black American ghetto and the French working-class borough in the post-Fordist age anchored by the three spatially inflected concepts of ghetto, hyperghetto and anti-ghetto. It concludes by stressing the role of the state in directing processes of seclusion at the top and at the bottom of the urban order, along a gradient from constraint to choice.
\end{abstract}

In jeder Epoche der kapitalistischen Gesellschaft, in der es zu einer raschen Transformation gekommen ist, haben die städtischen Armen die entscheidende Rolle zur Aufdeckung kollektiver Not gespielt. Sie waren der Auslöser für Innovationen im Bereich der Sozialforschung, für die Kontrolle sozialer Klassen und staatliche Eingriffe. Das Anwachsen der ,gefährlichen Klassen“ im Herzen von Paris zur Zeit der Restauration, die Bedrohung durch die „Gelegenheitsarbeiter“ im viktorianischen London, der Ansturm der „mobilen Ansammlungen" mittelloser Immigranten im Chicago der Jahrhundertwende, sie alle haben neue Formen der Sozialforschung und der sozialen Interventionen hervorgebracht.

In einem Kapitel mit dem Titel „Stadt der Furcht“ erwähnt Peter Hall, dass die moderne Stadtplanung, Stadtsoziologie und Sozialpolitik in erheblichem Maße durch eine komplexe emotionale Reaktion der Mittel- und Oberschicht aufgekommen sei. Teils aus Mitleid, teils aus Angst und Abneigung zielten sie darauf $a b$, etwas an der sie umgebenden städtischen Ungleichheit zu ändern (vgl. Hall, 1988:364). Die jüngste Jahrhundertwende macht da keine Ausnahme. Während des vergangenen Jahrzehnts haben das Wiederaufleben oder die Persistenz von Armut, sozialer Not, ethnisch motivierten Konflikten, Straßenkriminalität, Gewalt im öffentlichen Raum und ihre Akkumulation in den Elendsvierteln der
Metropolen zu der Frontlinie zwischen praktisch-politischen und wissenschaftlichen Debatten zurückgeführt. In Europa wie in Süd- und Nordamerika sind Staatschefs, Politikexperten und Aktivisten zunehmend beunruhigt über die „Unordnung", die sich in und um expandierende Gebiete des städtischen Verfalls herum zusammenbraut, und bestrebt, dieses „Chaos“ einzudämmen. Wie können SozialwissenschaftlerInnen diese Entwicklung deuten?

Im folgenden Beitrag wird die räumliche Abschließung oder Ausschließung als eine Technik zur Handhabung von Problemen und Problemgebieten untersucht. In einem ersten Schritt wird ein rudimentäres Bezugssystem entworfen, um die Instrumentalisierung des Raumes als ein Medium der sozialen Abschließung und Kontrolle in der Stadt analysieren zu können. In einem zweiten Schritt wird dieses Schema zur Präsentation der divergierenden Entwicklungslinien des afroamerikanischen Ghettos und des französischen Stadtviertels der Arbeiterklasse im postfordistischen Zeitalter genutzt. Die komprimierte Analyse ist in den drei räumlichen Konzepten des Ghettos, Hyperghettos und des Anti-Ghettos verankert, die im dritten Abschnitt des Beitrages dargestellt wird. Diese vergleichende Darstellung wird wiederum dazu dienen, die paradoxen Vorteile der Ghettoisierung zu betonen und die 
überragende Rolle des Staates in der sozialen Produktion und räumlichen Verteilung urbaner Marginalität herauszustellen.

\section{Ansätze einer sozioräumlichen Abschließung}

Zuerst wird eine Art analytische Erzählung skizziert, eine Geschichte der Dimensionen und Mechanismen, die die Untersuchung der sich wandelnden Formen städtischer Ungleichheit und Marginalität zu Beginn des neuen Jahrhunderts erleichtert sowie die Analyse dessen, was sie für die geschaffene Umwelt und indirekt für das berufliche Engagement und die Reflexion darüber implizieren. Das tiefere theoretische Rätsel, das zur empirischen Analyse der konkreten Manifestationen der Marginalität anregt, wie sie in Urban Outcasts (vgl. Wacquant, 2008) und Punishing the Poor (vgl. Wacquant, 2009a) dargelegt wurden, ist die Nutzung des Raumes als Produkt und Medium der Macht (vgl. auch Wacquant, 2009b). In dieser Hinsicht sind Ghetto, Hyperghetto und Anti-Ghetto, ebenso wie das Gefängnis, vier von zahlreichen Konfigurationen eines allgemeinen Prozesses, der sich als sozioräumliche Abschließung bezeichnen lässt. So gilt es zunächst, dieses abstrakte Konzept zu erklären und darauf aufbauend die Ghettoisierung sowie verwandte Formen zu lokalisieren und im analytischen Gitternetz zu fixieren.

Sozioräumliche Abschließung ist der Prozess, bei dem bestimmte soziale Kategorien und Aktivitäten in einen reservierten und abgegrenzten Quadranten eines physischen und sozialen Raumes eingesperrt, eingeschlossen und isoliert werden. Das englische Verb to seclude geht auf das Jahr 1451 zurück und leitet sich vom lateinischen Verb secludere ab, was so viel wie abschließen, isolieren und einengen bedeutet ${ }^{1}$. Sozioräumliche Abschließung kann Bevölkerungsgruppen betreffen (zum Beispiel MigrantInnen oder religiöse Sekten), Institutionen (solche wie medizinische Einrichtungen, Sport- und Gesellschaftsvereine, Sex-Shops) sowie Aktivitäten (Schulbesuch, Drogenhandel, Müllverbrennung). Sie lässt sich ferner nach dem Setting spezifizieren: Rurale sozioräumliche Abschließung erfolgt auf dem Land, während urbane sozioräumliche Abschließung in und um die Stadt herum gestaltet wird - ein Milieu, das nicht so sehr durch „Größe, Dichte und Heterogenität“ gekennzeichnet werden sollte, wie es im klassischen Stil der Chicagoer Schule nach Louis Wirth (1938) der Fall ist, sondern viel-

\footnotetext{
${ }^{1}$ Im Oxford English Dictionary (zweite Auflage, 1989) werden dem Verb to seclude sechs Bedeutungen zugewiesen, wovon die ersten beiden für den vorliegenden Beitrag relevant sind: „To shut, to enclose or confine so as to prevent access or influence from without. Also, to enclose or confine (a material thing) in a separate place“ „In wider sense: To remove or guard from public view; to withdraw from opportunities of social intercourse." Die erweiterte Semantik des Begriffs beinhaltet demnach auch Ausschließung und Ausweisung. Im mittelalterlichen Mittelenglisch bedeutete to seclude, jemanden von einem Privileg auszuschließen oder ihn zu entwürdigen.
}

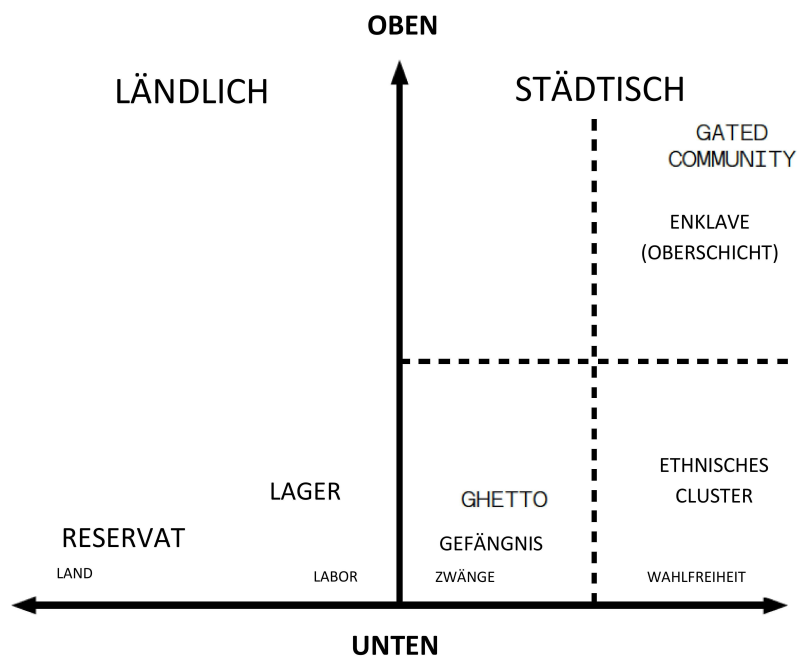

Abb. 1. Verschiedene Formen räumlicher Abschließung. Quelle: Eigener Entwurf

mehr durch die räumliche Akkumulation und intensive Anhäufung verschiedener Formen des Kapitals - ökonomisches, kulturelles, soziales und symbolisches - die den Schemata von Pierre Bourdieu (1986) und Max Weber (1921/1958) folgend - von einer administrativen Maschinerie gefördert werden.

Bei jeder Anordnung können Formen sozioräumlicher Aus- oder Abschließung entlang zweier Dimensionen zugewiesen werden (vgl. Abbildung 1). Die erste Dimension gibt Auskunft über die Ebene der sozialen Hierarchie. Sie deutet an, ob diese Hierarchie auf Klasse (Marktkapazität), Ethnizität oder auf einem Prestige-Ranking von Plätzen basiert - typischerweise sind diese fest korreliert, so dass sich an der Argumentation nichts fundamental ändert. Die Hierarchie kann entweder als ein Kontinuum behandelt werden oder - der Klarheit wegen - als eine Zweiteilung in eine Abschließung an der Spitze der Gesellschaft und eine am Boden. Die zweite Dimension gibt Auskunft darüber, ob die Abschließung gewählt ist, oder ob sie erzwungen ist. Im ersten Fall wird sie von einer internen Übereinstimmung angetrieben und gefestigt, im zweiten Fall von einer externen Feindseligkeit.

Diese idealtypischen Formen sozioräumlicher AbschlieBung können dann in dem zweidimensionalen Raum verortet werden, der von den beiden Achsen aufgespannt wird (siehe Abbildung 1): gewählt versus auferlegt, Spitze versus Boden. Im oberen rechten Quadranten befinden sich all jene, die ihre Isolation gewählt haben und Privatheit anstreben, die unter Ihresgleichen bleiben möchten und erniedrigte Bevölkerungsgruppen und zwielichtige Aktivitäten meiden. Diese Selbst-Exklusion an der Spitze, von einer innerkollektiven Orientierung angetrieben, wird von urbanen Elite-Enklaven oder traditionellen Stadtvierteln der Oberschicht repräsentiert (solche, wie sie vom Pariser Großbürgertum errichtet, aggressiv verteidigt und von Pinçon und 
Pinçon-Charlot (2007) beschrieben wurden) und, in einer übertriebenen Form, von den gated communities, die sich in den letzten beiden Jahrzehnten explosionsartig an der Spitze der sozialen und räumlichen Hierarchie vermehrt haben (wo gewollte Isolation durch physische Begrenzungen konkretisiert wird, wo ein Zaun mit einem Tor und Wachmännern Überwachung und Schutz garantiert), um einen Zufluchtsort der sozialen Homogenität und der Sicherheit vor Kriminalität zu bieten sowie die Vorzüge einer Mitgliedschaft in einer privilegierten Gemeinde an einem sozialen Gunstraum (Blakely and Snyder, 1999; Atkinson and Blandy, 2006). Während sich also an der Spitze der sozialen Hierarchie vornehme Aktivitäten mächtiger Personen ausmachen lassen, die mit dem nötigen ökonomischen und symbolischen Kapital ausgestattet sind, um andere Menschen auszuschließen und sich selbst einzuschließen, sind am Boden der sozialen Hierarchie unedle Aktivitäten ,verdorbener“ Personen zu beobachten, die weder ökonomisches noch kulturelles Kapital besitzen, mit anderen Worten, die Besitzlosen und Entwürdigten.

Die beiden wesentlichen Formen, die zur sozioräumlichen Abschließung am Boden der urbanen Hierarchie führen, sind das Ghetto und das ethnische Cluster. Diese Formen dürfen nicht verwechselt werden, da sie an den beiden entgegengesetzten Endpunkten des Kontinuums von Auflage versus Wahl einzuordnen sind und unterschiedliche Funktionen erfüllen. In Richard Sennetts Werk „Flesh and Stone“ (1994), in dem er die Entstehung des ersten Ghettos im Venedig des 16. Jahrhunderts nachzeichnet, verwendet er einen sehr treffenden Ausdruck, um dessen Funktion zu veranschaulichen (vgl. 1994:228-237). Er bezeichnet das Ghetto als ein städtisches Kondom, da seine Beschaffenheit einerseits die Penetration der christlichen Stadt durch die Juden ermöglichte (im Finanzwesen, im Handel und im kulturellen Bereich, die für den Erfolg des Hofes entscheidend waren, wurden sie gebraucht), andererseits jedoch intimen Kontakt mit ihnen verhinderte (der jüdische Körper galt als korrupt und korrumpierend, als Überträger von Krankheiten und als Sakrileg). Das Ghetto entwickelte sich zu einer sozioräumlichen Einrichtung, welche gleichzeitig die wirtschaftliche Ausbeutung und soziale Ausgrenzung der Paria ermöglichte. Tagsüber schwärmten die Juden in die Stadt aus, um ihren wirtschaftlichen Pflichten nachzukommen, abends kehrten sie unter Androhung schwerer Strafen in das für sie vorgesehene, abgeschlossene Viertel zurück. Wenn sie sich außerhalb der Mauern ihres Ghettos bewegten, waren sie verpflichtet, bestimmte Kleidungsstücke zu tragen (beispielsweise einen gelben, spitzen Hut), damit die Christen sie identifizieren und meiden konnten. Dieselbe räumliche Einrichtung wurde vier Jahrhunderte später im Nordosten und im mittleren Westen der Vereinigten Staaten neu erfunden, als Afroamerikaner aus dem Süden in die expandierenden Industriestädte des Nordens migrierten, die ihre Arbeitskraft wollten, jedoch kein Zusammenleben mit dort ansässigen Weißen, um „soziale Gleichheit“ zwischen den so genannten Rassen und die verabscheute „Rassenmischung“ zu vermeiden (vgl. Spe- ar, 1968; Osofky, 1971; Phillips, 1999). Die Hautfarbe zeigte hier die Mitgliedschaft in einer inferioren ,ethnorassischen“ Kategorie an, die es zu meiden galt. Die scharf abgegrenzte Bronzeville diente hier als Arbeitskräftepool und auch als Container kontaminierender Körper.

In der Zwischenkriegszeit beging die Chicagoer Schule, die in den USA die soziologische Erforschung der Stadt aus der Taufe hob, den Kardinalsfehler, die von Wellen irischer, italienischer und polnischer Immigranten geformten stationären und institutionellen Ansammlungen pauschal mit einem nicht genauer definierten Begriff des "Ghettos“ zu etikettieren, das sowohl jüdische Bezirke als auch den so genannten „Schwarzen Gürtel“ abdeckte. In Anlehnung an Louis Wirth, einem der Begründer der Stadtökologie (vgl. 1928), lässt sich diese misslungene Einordnung aus zwei Gründen als Wirthschen Irrtum bezeichnen. Erstens hat es in den USA, wie es Wirth in seinem Klassiker ,The Ghetto" unabsichtlich belegt, nie ein jüdisches Ghetto gegeben. Es hat lediglich als „Geisteszustand“ existiert, welcher ein subjektives Wir-Gefühl und eine kulturelle Orientierung darstellt und sich stark von einer sozioräumlichen Einrichtung unterscheidet. Zweitens wurden in den afroamerikanischen Enklaven, im Gegensatz zum Schwarzen Gürtel, alle AfroamerikanerInnen, ganz gleich welcher Klasse, durch eine Kombination aus rechtlicher Beeinflussung, Einschüchterung auf der Straße und kollektiver Gewalt gezwungen, dort zu wohnen, während die weißen ethnischen Cluster in ihrer Zusammensetzung gemischt waren, mobil in ihrer Verortung und nur eine Minderheit der jeweiligen Migrantenbevölkerungen beinhalteten, die dort hauptsächlich wegen Klassenbeschränkungen und kultureller Identität wohnten.

In architektonischen Begriffen kann das (weiße) Cluster als Brücke dargestellt werden, das (schwarze) Ghetto hingegen als Mauer. Die Brücke ist ein Mechanismus flexibler und temporärer Ausschließung innerhalb eines durchlässigen Umrisses, die als ein Akklimatisierungsraum und als eine Zwischenstation auf dem Weg zu einer sozioräumlichen Integration in die weitere Gesellschaft fungiert. Die Mauer ist hingegen ein Mittel zur inflexiblen und permanenten Ausschließung innerhalb einer Spitztüte (undurchlässiger Sack), die der dauerhaften Isolierung und Dissimilation der Bevölkerung dient (bis sie zusammenbricht). Das ethnische Cluster und das Ghetto haben somit voneinander abweichende Strukturen und erfüllen unterschiedliche Funktionen, weswegen es ein fundamentaler soziologischer Fehler ist, sie in einen Topf zu werfen.

Um den Unterschied zwischen Ghetto und ethnischem Cluster zu begreifen, ist es hilfreich, am Boden des Diagramms zur sozioräumlichen Ausschließung eine dritte Institution der auferlegten Einsperrung einzutragen: das Gefängnis. Das Gefängnis nutzt die physische Einschränkung der Mauern und die Macht der Aufseher, um die Gefangenen zu segregieren. Letztere stellen eine diskreditierte Kategorie dar, deren Rechte und Kontakte zu „Outsidern“ wegen eines Verstoßes gegen einen sozialen Verhaltensstandard amputiert 
sind. Das Gefängnis ist eine Art gerichtliches Ghetto, in dem Insassen eine Parallelgesellschaft und eine eigene Kultur entwickeln als Antwort auf eine erzwungene Isolation und die Entbehrungen, die mit ihr einhergehen. Umgekehrt könnten wir das Ghetto als ein ,ethnorassisches Gefängnis“ bezeichnen, das eine nicht akzeptierte Bevölkerung in einen speziellen Bezirk einsperrt, in dem sie gezwungen ist, eine separate Lebenssphäre als Reaktion auf eine räumliche Beschränkung und eine soziale Verbannung zu entwickeln. Sobald wir die strukturelle und funktionale Verwandtschaft zwischen Ghetto und Gefängnis begreifen (die Nähe im Diagramm der Formen sozioräumlicher Ausschließung deutet darauf hin), verstehen wir, warum der Kollaps der Ghettos nach den Aufständen der 1960er Jahre als ein Substitut für eine als unehrenhaft, mittellos und gefährlich erachtete Bevölkerungsgruppe zu einem Anwachsen der Gefängnisse geführt hat (vgl. Wacquant, 2001 und 2009a).

Das Ghetto ist per Definition ein urbanes „Getier“. Im ländlichen Raum lassen sich die Modalitäten der räumlichen Ausschließung danach differenzieren, ob die untergeordnete Bevölkerung sich bewegen muss, um ihre Arbeitskraft anzubieten, oder ob sie bewegt wird, um Land abzugeben, das sie besetzt. In den Fällen, in denen die dominante Gruppe es nicht wünscht oder nicht in der Lage ist, die Untergebenen zu beschäftigen, jedoch darauf abzielt, sich ihr Land anzueignen, wie es bei kolonialen Konflikten gegeben war, beobachten wir häufig das Aufkommen eines Reservats. Das ist die Geschichte der Native Americans: Aus einer Vielfalt von demographischen, kulturellen und politischen Gründen stellten sie kein geeignetes Angebot an Arbeitskräften dar, aber sie besetzten wertvolles Land, das für das koloniale Projekt agrarischer Expansion unverzichtbar war. Daher wurde die sozioräumliche Abschließung dergestalt durchgeführt, dass sie in abgegrenzte Gebiete umgesiedelt wurden. Die Bedrohung, die sie darstellten, wurde so neutralisiert (vgl. Cornell, 1988). (Was im Übrigen auch auf andere indigene Völker zutrifft, z.B. die Kanaken in Neukaledonien, vgl. Liauzu, 2007). Wenn wir uns auf der Achse Land/Arbeit nach rechts bewegen, finden wir intermediäre Konstellationen, die aufkommen, um die Arbeitskraft der untergeordneten Bevölkerung zu gewährleisten, während sie davon abgehalten werden, in die Stadt zu kommen. Denn eine umfassende Urbanisierung würde ihre Reproduktionskosten erhöhen und Druck zur Durchmischung generieren (was die ,ethnorassische Reinheit" und Hierarchie untergraben würde). Bei solchen Szenarien kommt es zur Herausbildung eines Lagers, von dem zwei Haupttypen existieren, das Arbeitslager für WanderarbeiterInnen (genauso wie für Sträflinge und politische Gefangene) und das Flüchtlingslager für politisch vertriebene Menschen (vgl. hierzu auch einige recht provokative Untersuchungen des Ethnologen Michel Agier, 1999, 2008).

Kurz zusammengefasst besteht der Vorteil des hier skizzierten Schemas darin, dass es uns erlaubt, die verschiedenen Formen der sozioräumlichen Abschließung in ein einziges analytisches Bezugssystem zu bringen und sie zusammen zu untersuchen, anstatt sie getrennt voneinander zu analysieren, so, als ob sie zu verschiedenen Arbeitsgebieten gehörten (Agrarwissenschaften, Stadtsoziologie, Kriminologie sowie Ethnologie der Klassen und Ethnizität) und unterschiedlichen Logiken folgten. Es geht Wesentliches durch die konventionelle Segmentierung der Forschung in Disziplinen verloren, bei der Raum genutzt wird, um Kategorien und Aktivitäten zu definieren und zu begrenzen.

Die Kategorie der Abschließung kann nicht nur dazu dienen, die räumlichen Erfahrungen verschiedener Bevölkerungsgruppen auf unterschiedlichen Ebenen der sozialen Struktur zu vergleichen und zu kontrastieren, sie kann auch genutzt werden, zu zeigen, wie dieselbe Bevölkerung durch eine Kombination räumlicher Vorrichtungen im Laufe der Zeit eingesperrt wird. Zum Beispiel ließe sich Südafrika in das Diagramm einfügen. Am linken Ende des Spektrums befänden sich die aus der kolonialen Ära unter dem Regime der „Segregation“ (1910-1948) übernommenen Reservate und später die Bantustans, die schwarzen Marionettenrepubliken, die 1958 von den weißen Machthabern erfunden wurden, um die Staatsbürgerschaft der Schwarzen räumlich und ethisch zu „externalisieren“. Näher zur Mitte des Diagramms hin ließen sich die Bergwerksgebiete lokalisieren, eine Variante des Arbeitslagers, mit denen rotierende ArbeitsmigrantInnen zum Nutzen der Rohstoffindustrie während des Booms der mineralischen Lagerstätten rekrutiert und fixiert wurden. Reservat und Bergwerkslager sollten zusammen den Prozess der Urbanisierung stoppen. Dies schlug jedoch fehl, so dass sich Schwarze massenhaft in Städten ansiedelten, wo sie dann in townships (Südafrikas Version des Ghettos) verbannt wurden. Dort brachten sie schrittweise die Ressourcen auf, um die Herrschaft der Weißen herauszufordern und schlussendlich zu überwinden. Unterdessen prosperierten an der Spitze der ökonomischen, ,ethnorassischen“ und urbanen Struktur die befestigten Elite-Enklaven und abgesonderten gated communities der Oberschicht, die während der Apartheid ausschließlich weiß waren und erst nach 1994 teilweise schwarz wurden (vgl. Murray, 2008).

Südafrika ist ein besonders ergiebiges Terrain zur Erforschung sozioräumlicher Abschließung, da die südafrikanische Gesellschaft die Verräumlichung der Herrschaft auf die Spitze getrieben hat. Vor allem in der Ära der Apartheid schuf der Staat eine enorme bürokratische Maschinerie und ein aufwendiges Regelwerk (vgl. Evans, 1997), um eine hohe Übereinstimmung zwischen symbolischem Raum (die rigide Einteilung der Gesellschaft in eine Hierarchie gegenseitig ausschließender offizieller ,ethnorassischer“ Kategorien), sozialem Raum (die Allokation effizienter Ressourcen zu diesen Kategorien) und physischem Raum (die autoritative Distribution der Bevölkerungen in Städte und ländliche Gebiete) durchzusetzen. In dieser Hinsicht unterscheidet sich Südafrika zum Beispiel von Brasilien, wo sich die ,ethnorassische" Einteilung und ihre Projektion auf den Raum durch Unschärfe auszeichnen. Dies deutet auf die Notwendigkeit vergleichender Studien hin, die sich mit der Frage 
beschäftigen, welche Kombinationen sozioräumlicher Ausschließung sich in welcher Gesellschaft entwickeln und aus welchen Gründen (vgl. den Beitrag von Rothfuß und Gerhard in diesem Heft).

\section{Struktur, Funktion und Schicksal des US-amerikanischen Ghettos}

Im Folgenden werden das Ghetto als eine Art der sozioterritorialen Abschließung und das Schicksal des USamerikanischen Ghettos nach der Bürgerrechtsbewegung als ein historisches Puzzle untersucht. Um ein Ghetto erzeugen zu können, bedarf es vier struktureller Elemente. Das erste ist ein Stigma: Ein Ghetto entsteht durch den Umgang mit einer Bevölkerung, die befleckt ist, die in den Augen der dominanten Kategorie besudelt (ist), so dass ein intimer Kontakt mit ihr unterbunden und verboten werden muss. Bei den Juden, die in den Ghettos Europas im Zeitalter der Renaissance lebten, war das Stigma religiös, verbunden mit der weit verbreiteten christlichen Überzeugung, dass Juden für den Tod Christi verantwortlich seien sowie Überträger ansteckender Krankheiten und Beförderer der Sittenlosigkeit darstellten (vgl. Stow, 1992). Bei den AfroamerikanerInnen, die in den Industriemetropolen während des fordistischen Zeitalters zwischen 1910 und 1970 lebten, war es der mit der Sklaverei assoziierte Makel, tagtäglich ausgearbeitet durch den religiösen und pseudo-wissenschaftlichen Glauben an die unterstellte Inferiorität und Bestialität der Schwarzen (Kolchin, 1993).

Das zweite strukturelle Element des Ghettos ist der Zwang: Wie bereits festgestellt, können Bevölkerungen als ein Ergebnis externer Auferlegung räumlich konzentriert werden, jedoch auch aus Affinität heraus, durch ausgewählte Selbst-Einschließung, die auf Klasse, Kultur oder Lebensstil basiert. Daher dürfen die beiden Szenarien nicht verwechselt werden: Um es noch einmal zu wiederholen, zu Beginn des 20. Jahrhunderts kamen die so genannten weißen Ethnien lose und temporär in gemischten ethnischen Clustern zusammen (Little Italy, Little Ireland, Germantown etc.), als Ergebnis einer Kombination aus Immigrantenerfahrung, Klassenposition und kultureller Sympathie; jedoch wurden sie nie gleichförmig gezwungen in einem fixierten und reservierten Territorium zu leben, in dem ausschließlich Mitglieder derselben Ethnien residierten, so wie es nach 1910 den Afroamerikanern in den Städten auferlegt wurde (vgl. Philpott, 1978; Lieberson, 1980).

Das dritte konstituierende Element des Ghettos ist die räumliche Abschließung: ein Ghetto entsteht, wenn stigmatisierte Personen in einem abgegrenzten Gebiet wohnen, in das sie gewaltsam zugeteilt wurden, so dass Kategorie und Territorium eine wechselseitige Implikation der ethnischen Homogenität und Ausschließlichkeit haben. Dieses Phänomen leitet über zum vierten und letzten konstituierenden Element des Ghettos, zum institutionellen Parallelismus. Da die stigmatisierte Bevölkerung unter Druck gesetzt wird, ausschließlich in dem für sie vorgesehenen Bezirk zu wohnen, entwickelt sie ein Netz an Institutionen, die die Institutionen der Gesellschaft, von der sie zurückgewiesen wird, verdoppeln und ersetzen. Daher bildeten die italienischen Juden der frühen Moderne, die genötigt waren, in einem vorgesehenen Judenviertel zu leben (bis ein Großteil von ihnen es dann bevorzugte, separat zu wohnen), innerhalb des Ghettos eine Vielzahl an kulturellen, ökonomischen und wohltätigen Organisationen heraus. Sie schufen eine selbsteingrenzende jüdische Stadt innerhalb der christlichen Stadt (vgl. Calimani, 1987), so wie die AfroamerikanerInnen, die in der Zwischenkriegszeit aus dem ländlichen Süden in die fordistischen Metropolen migrierten, eine ,schwarze innerhalb der weißen Stadt" konstruierten, um jene kollektiven Bedürfnisse zu befriedigen, die aufgrund der weißen Exklusion nicht erfüllt worden waren (Drake and Cayton, 1945, 1963).

Dies führt uns nun zu den beiden widersprüchlichen, unvereinbaren Rollen, die das Ghetto an den beiden Enden der Beziehungen ,ethnorassischer“ Herrschaft spielt. Historiker der jüdischen Diaspora in Europa, Ethnologen der Kastenregime in Asien und Afrika und Soziologen der Afroamerikaner im industrialisierten Amerika haben die Rollen dokumentiert, paradoxerweise jedoch nicht begriffen. Vom externen Standpunkt des Beherrschenden aus erkennt man leicht, dass das Ghetto ein Instrument zur Beherrschung darstellt, da es den Lebensraum und die Lebenschancen der eingeschlossenen Kategorie scharf begrenzt. Aus der Innenperspektive heraus - und dies ist ebenso wichtig - ist das Ghetto jedoch ein robuster sozioräumlicher Schild, ein beschützender Puffer, der eine unverwechselbare Lebenswelt im Innern kreiert, in welcher der Dominierte, fernab von einem direkten Kontakt mit dem Dominierenden, regelrecht aufatmen kann (vgl. Wacquant, 2004).

Vom Standpunkt der Juden in der frühmodernen europäischen Stadt und von jenem der Afroamerikaner in der fordistischen Metropole gab ihnen die Ghettoisierung eine separate und geschützte Sphäre, die Interaktionen von Angesicht zu Angesicht und Spannungen mit den Dominierenden verringerte. Außerdem befähigte sie das Ghetto, ökonomisches Kapital zu akkumulieren, verschiedene Formen kulturellen Kapitals zu entwickeln und soziales Kapital anzuhäufen, das nötig war, um gegebenenfalls das Stigma und der erlittene Ausschluß zu überwinden. Die Wirksamkeit des Ghettos als ein Gerüst der sozialen Kohäsion und eine Maschine der kulturellen Produktion ermöglicht es seinen BewohnerInnen, die ihnen kollektiv zugewiesene negative Symbolik umzukehren - wie sich am Aufkommen des Slogans „Black is beautiful" mit den zeitgleichen Wellen der Aufstände in den 1960er Jahren zeigte - und gegebenenfalls die sozioräumliche Einrichtung anzugreifen, die sie beschränkt und eingrenzt (Wacquant, 2006).

Der anklägerische Ansatz, der gemeinhin von Sozialwissenschaftlern angenommen wird, hat die Erkenntnis verhindert, dass das Ghetto eine zweiseitige Einrichtung ist: Es ist 
sowohl ein Instrument der Unterordnung als auch ein Raum des Schutzes, der Vereinigung und der Kohäsion. Wir müssen uns die verdeckten, wenig eingängigen Leistungen der Ghettoisierung vor Augen führen, die einer untergeordneten „ethnorassischen“ Kategorie ein Vehikel der Selbstorganisation und Mobilisierung anbietet und es ihnen folglich ermöglicht, ihre „Macht von unten“ wirksam einzusetzen. Das ist der Grund, weswegen Ghettos strukturell instabile Formationen sind, die eine begrenzte Haltbarkeitsdauer haben (auBerhalb von staatlich basierten Formationen): Sie pflanzen und züchten die Saat ihrer eigenen Zerstörung, indem sie die Untergeordneten stärken und hervorstechende und festumrissene ,ethnorassische“ Grenzen ziehen, die angegriffen werden können - wohingegen andere Konfigurationen der Herrschaft, wie zum Beispiel die unscharfe und sich auflösende Segregation in der Art, wie sie postkoloniale MigrantInnen in West-Europa oder dunkelhäutige BrasilianerInnen erleben, es entwürdigten und mittellosen Bevölkerungen erschweren, als eine Gruppe zusammenzukommen und ihre Marginalisierung zu reflektieren und zu bekämpfen.

Was passierte mit Amerikas schwarzem Ghetto nach den Umbrüchen der 1960er Jahre? Sofern man das Ghetto nicht nur als einen segregierten Ort, als einen Bezirk heruntergekommener Bausubstanz und sozialen Verfalls sowie als eine ,üble“ Nachbarschaft begreift, die alle Formen sozialer Pathologien beinhaltet, darunter Lasterhaftigkeit und Gewalt (wozu es führen kann, nachdem es auseinanderbricht); wenn man es als eine besondere sozioräumliche Einrichtung versteht, die darauf abzielt, ökonomische Ausbeutung und soziale Ausgrenzung zu bewirken, dann lassen sich die Gründe der brutalen Implosion von Amerikas Bronzeville nach dem Höhepunkt der Bürgerrechtsbewegung ausmachen. Das dunkle Ghetto brach auseinander und zerbröckelte in sich, da es unter dem Druck dreier konvergierender Kräfte stand. Die erste Kraft ist ökonomisch: Es handelt sich um den Übergang von einer industriell-fordistischen Wirtschaft, bei der die Produktion innerhalb der Stadt angesiedelt war und einen großen Pool ungelernter Arbeitskräfte verlangte, zu einer dezentralisierten dienstleistungsbasierten Wirtschaft, in der Automatisierung, globale Standortwechsel und eine nochmalige Unterschichtimmigration urbane schwarze Arbeitskräfte überflüssig machten. Die zweite Kraft ist demographisch und politisch: Sie soll hier als die große weiße Migration bezeichnet werden. Historiker haben exzellente Werke zur großen schwarzen Migration aus dem Süden in die nördliche Stadt während der Zwischenkriegszeit veröffentlicht (vgl. Trotter Jr., 1991; Gregory, 2007), eine verständliche Darstellung der massiven weißen Abwanderung in die Vorstädte als Reaktion auf den schwarzen Einzug sowie eine Untersuchung ihrer Auswirkungen auf Amerikas Gesellschaft, Kultur und Politik lassen jedoch noch auf sich warten.

In den 1960er und 1970er Jahren flohen Millionen Weiße in die Vorstädte, um dort die soziale und räumliche Distanz zu den Schwarzen aus dem Süden wiederherzustellen, die in die Metropolen strömten. Dieser riesige Bevölkerungstrans- fer verlagerte den Wählerschwerpunkt des Landes von den Innenstädten in die Vorstädte, wodurch der politische Einfluss der innerstädtischen, im ökonomischen Bereich ohnehin schon marginalisierten Bevölkerung zusätzlich verringert wurde. Der Transfer verursachte auch die Finanzkrise der Städte in den 1970er Jahren, die von den politischen Eliten als Vorwand genutzt wurde, um Programme für die Armen zu kürzen und Stadtpolitik auf die Beschaffung von Unternehmensbetreuung und Annehmlichkeiten für die Mittelschicht auszurichten. Die dritte Kraft, die das Ghetto zum Einsturz brachte, ist die erfolgreiche schwarze Mobilisierung gegen weiße Herrschaft in Form der Bürgerrechtsbewegung und ihres radikalen Ablegers der Black-Power-Bewegung, welche die sozioräumliche Abschließung und ökonomische Ungleichheit in der Metropole massiv in Frage stellten.

Diese drei Faktoren trafen zusammen und bewirkten die Implosion des Ghettos, die einer dualen Konstellation, bestehend aus dem Hyperghetto und den schwarzen Mittelschichtsvorstädten, den Weg ebnete. Die Massenflucht der Weißen in die Vorstädte schuf Lücken, in denen sich die afroamerikanische Mittelschicht einrichten und in segregierten Bezirken wachsen konnte, angrenzend an das historische Ghetto und getrennt von beiden, von den Weißen und von der schwarzen Unterschicht, die in der kollabierenden Bronzeville gefangen war. Die 1970er Jahre initiierten daher einen gepaarten Prozess von sozialer Differenzierung und räumlicher Trennung der schwarzen Bevölkerung, bei dem die Abschließung der AfroamerikanerInnen in der Stadt fortgesetzt wurde, jedoch durch eine sich gabelförmig teilende Einrichtung, die in zwei unterschiedliche urbane Container gegliedert ist: Das Hyperghetto für die prekären Fraktionen der Arbeiterklasse und die segregierten Viertel, physisch und sozial getrennt vom Hyperghetto für die Mittel- und Oberschichten.

Drei Eigenschaften unterscheiden das Hyperghetto vom kommunalen Ghetto, das in den 1910er Jahren aufkam, um 1950 herum aufblühte und in den späten 1960er Jahren zusammenfiel. Erstens fehlt es insofern an einer ökonomischen Funktion, als die Arbeitskraft, die es beinhaltet, überflüssig geworden ist. Zweitens hat die ökonomische Überflüssigkeit zu einer sozialen Ent-Differenzierung und zu einem Auflösungsprozess der schwarzen Bourgeoisie geführt, so dass das Hyperghetto durch ,Rasse und Klasse doppelt segregiert ist. Drittens und infolgedessen ist es seiner eingewachsenen Institutionen beraubt worden, die den Puffer gegen die Beherrschung und den Rahmen des Alltagslebens bereitstellten. Die kommunalen Institutionen der historischen Bronzeville sind durch die staatlichen Bürokratien der Sozialkontrolle ersetzt worden: Wohlfahrt wurde zu workfare, öffentliche Schulen wurden zu Aufsichtsanstalten herabgesetzt und hinzu kamen eine aggressive Polizei, Strafgerichtshöfe sowie ein offensives Gefängnissystem mit seinen Ausweitungen wie zum Beispiel den Bewährungshelfern (vgl. Wacquant, 2009a). Zusammengefasst ist das Hyperghetto eine sozioräumliche Einrichtung, die auf unverhüllte Exklusion abzielt, die die schwarze Community entlang von Klassenlinien aufsplittert 
und keine der kollektiven Absicherungen und Nebenvorteile der Ghettoisierung bietet.

\section{Ausschließung in Frankreich: Vom Quartier der Arbeiterklasse zum Anti-Ghetto}

An dieser Stelle wird nun der Atlantik überquert, um den Ablauf des Niedergangs der Peripherie der französischen Stadt ab Mitte der 1970er Jahre nachzuzeichnen. Eingangs sei betont, dass diese Territorien der Arbeiterklasse in den Medien und politischen Debatten gemeinhin als „ImmigrantenGhettos" porträtiert und verunglimpft wurden, während sie in Wirklichkeit gemischte Areale darstellen, sowohl bezüglich der Berufe als auch der Ethnizität (vgl. Stébé, 2007 für Frankreich und Musterd et al., 2006 für ein europäisches Panorama). Von ein paar lokalen Ausnahmen abgesehen, umfassen Frankreichs Unterschicht-banlieues Bevölkerungen, die mehrheitlich französisch sind. Sie vermischen BewohnerInnen von einem Dutzend bis hin zu fünf Dutzend verschiedenen Nationalitäten. Historisch waren sie in der Industriearbeit verankert und gekennzeichnet durch die feste Integration in die Welt der Akkordarbeit, kommunaler Dienste, Fabrikarbeitermilieu und des Familienlebens. Diese enge Passform von Produktionsstätte, lokalem Staat und Gemeinde fiel unter dem Druck der Deindustrialisierung, der Massenarbeitslosigkeit und der Universalisierung der Schulbildung als einer Form des Zugriffs auf wertgeschätzte soziale Positionen in sich zusammen.

Im Folgenden wird die staatliche Politik im Hinblick auf die Wohnformen der Einkommensschwachen fokussiert, denn dieses Phänomen ist für Architekten, Stadtplaner und Geographen von besonderem Interesse. Schematisch dargestellt, sind französische Regierungen während der letzten drei Jahrzehnte von einer Finanzierung des sozialen Wohnungsbaus, die für die Arbeiterklasse vorgesehen waren, zu einer Bereitstellung von Subventionen für individuelle Haushalte übergegangen, um ihnen beim Wechsel in den Markt der Einfamilienhäuser zu helfen. Diese staatlichen Subventionen befähigten untere Mittelschichtfamilien, die Großwohnsiedlungen des öffentlichen Sektors zu verlassen und in individuelle Häuser in privaten Parzellen zu migrieren. Dieses „,creaming“ der materiell stabilen Haushalte hat in den großen Immobilien nur die Familien der Arbeiterklasse zurückgelassen, die durch einen dramatischen Anstieg der Arbeitslosigkeit und eine unerbittliche Verbreitung unsicherer Arbeitsplätze unterhöhlt wurden. Das Ergebnis ist der physische Verfall, die ökonomische Verelendung und die öffentliche Diffamierung der städtischen Peripherie, die nun allgemein als elende Löcher wahrgenommen werden, in denen nur die von der Gesellschaft Ausgestoßenen ertragen zu leben (vgl. Wacquant, 2007).

Es stellt sich somit folgende Frage: Ist nun die urbane Arbeiterklasse in Richtung Ghetto abgedriftet? Die Antwort auf diese Frage fällt eindeutig negativ aus. Bezüglich empiri- scher Details sei auf Urban Outcasts (vgl. Wacquant, 2008) verwiesen. An dieser Stelle seien vier Trends genannt, die belegen, dass die verunglimpften Bezirke der europäischen Metropolen sich in die entgegengesetzte Richtung entwickelt haben, so dass man sie als Anti-Ghettos bezeichnen kann (wenn man diesen Begriff weiterhin verwenden möchte, was weder angebracht noch nützlich erscheint).

Zunächst bedeutet Ghettoisierung, dass Mitglieder einer ab- bzw. ausgestoßenen Kategorie gezwungen werden, in einem abgeschotteten, für sie vorgesehenen Raum zu leben, was zu einer zunehmenden ethnischen Homogenität führt. Die aufgegebenen Bezirke des so genannten Roten Gürtels von Paris und die verwandten französischen banlieues sind in dieser Hinsicht während der letzten beiden Jahrzehnte weniger homogen geworden.

Ein zweiter Indikator der Ghettoisierung ist eine zunehmende organisatorische Dichte: Eine stigmatisierte Kategorie, abkommandiert in eine vorgesehene Enklave, wird sie mit eigenen Institutionen füllen, was sich an der wachsenden und zunehmenden Differenzierung informeller und formeller Organisationen belegen lässt. Die Territorien der Arbeiterklasse in Westeuropa, von den britischen midlands, über das deutsche Ruhrgebiet, bis hin zu den quartieri degradati der norditalienischen Städte, bezeugen das genaue Gegenteil: Den Rückgang und den Tod lokaler Organisationen, vor allem jener, die der Industriearbeiterklasse sowohl im Bereich der Arbeit als auch in benachbarten Bereichen Hilfe anbieten. In der Mehrzahl der Fälle handelt es sich bei den Vereinigungen, die dort gegenwärtig präsent sind, um direkte oder indirekte Ableger des Staates, nämlich öffentliche Beamtenapparate und Gemeindebehörden, die auf staatliche Gelder angewiesen sind.

Wie bereits angedeutet, handelt es sich bei einem Ghetto drittens um eine kulturelle Fusionsmaschine, die das Aufkommen eines geteilten Idioms der Identifikation und des Aufstellens von Forderungen begünstigt, das die verschiedenen Komponenten der stigmatisierten Bevölkerung umfasst. Daher unterhöhlte die Ghettoisierung im Falle der Juden die Unterscheidung zwischen Aschkenasen und Sepharden, und es ließ in ähnlicher Weise die Unterschiede zwischen Schwarzen und Mulatten verschmelzen, die es im Falle der AfroamerikanerInnen bis in die 1920er Jahre angestrebt hatten, als eigene Kategorie anerkannt zu werden. Die sich verringernden Territorien der Arbeiterklasse in Westeuropa weichen hier in ihrem Misslingen, eine gemeinsame Identität zu entwickeln, erneut von diesem Schema ab. Seit mittlerweile dreißig Jahren haben Politiker und Journalisten angekündigt, dass „,das Ghetto“ in Frankreich angekommen sei. Sie haben mit angsterfüllten Worten die unterstellte „Amerikanisierung" der Stadt verurteilt. In Wirklichkeit bleiben die BewohnerInnen der banlieues entlang der Kategorien von Klasse, Nationalität, Ethnizität (innerhalb der Nationalität), Alter und Generation tief gespalten. Das einzige symbolische Etikett, das sie teilen, ist das Stigma, in einem Bezirk des Verfalls zu wohnen. 
Ein viertes Charakteristikum des Ghettos ist die Unpassierbarkeit seiner Grenzen, da es darauf abzielt, alle Mitglieder einer anvisierten Kategorie einzuschließen, unabhängig von deren Einkommen und sozialen Rang. Die jüdischen Ghettos des vorrevolutionären Europas, wie jenes in Frankfurt und Prag, hielten reiche und arme Juden zusammen; Chicagos Bronzeville schloss in seiner Umfassung alle schwarzen Klassen ein, von den Bettelarmen bis hin zu den Vermögenden, von den Kriminellen bis hin zu den Salonfähigen, selbst wenn sich diese innerhalb des Schwarzen Gürtels zu unterschiedlichen Parzellen hingezogen fühlten. Nicht so in der europäischen Stadtperipherie, wo die Familien postkolonialer MigrantInnen, die in der Klassenstruktur aufsteigen durch das Bildungssystem, geringfügiges Unternehmertum, Lohnarbeit (oft im öffentlichen Sektor) oder Eheschließung - sofort aus ihrer alten Wohngegend wegziehen. Und wie bei den Latinos und den so genannten europäischen Ethnien in den Vereinigten Staaten führt aufwärts gerichtete soziale Mobilität auch in Europa zu räumlicher Zerstreuung und sozialer Integration.

In der Summe werden die abnehmenden Unterschichtbezirke der französischen und generell der europäischen Metropolen ethnisch heterogener und organisatorisch weniger dicht; ihre Grenzen sind porös. Auch haben sie es nicht geschafft, eine geteilte kulturelle Identität $\mathrm{zu}$ formen. In vierfacher Hinsicht gehorchen sie einer genau entgegengesetzten sozioräumlichen Dynamik zu jener, die das Ghetto produziert. Deshalb sollten sie als Anti-Ghettos bezeichnet werden, eine Provokation, die an die Advokaten der modischen „Amerikanisierungsthese“ der europäischen Stadt adressiert ist. Denn bei genauer Betrachtung erweist sich der Begriff der Ghettoisierung als fundamental unangemessen, um den Prozess der städtischen Abschließung in Westeuropa, hier vorgestellt v.a. in Frankreich, zu Beginn des 21. Jahrhunderts verstehbar zu machen.

\section{Zusammenführung: Das letzte Element in der Gleichung städtischer Abschließung}

Um die Logik der Verbannung in der amerikanischen und europäischen Metropole zu verstehen, muss das Kriterium spezifiziert werden, nach dem bestimmte Bevölkerungsgruppen aussortiert und auf den „Bodensatz“ des stratifizierten Ortssystems platziert werden, das die Stadt ausmacht. Auf der amerikanischen Seite ist das Einhegen im dunklen Ghetto durch die Kategorie Ethnizität determiniert - was in dieser besonderen Variante der nicht aufgehobenen Ethnizität gemeinhin als „Rasse“ bezeichnet wird - später durch Klasse moduliert wird (mit dem Aufkommen des Duetts, bestehend aus Hyperghetto und segregiertem schwarzen Mittelklasse-Satelliten) und unverwechselbar intensiviert durch den Staat mit Hilfe seiner Wirtschafts-, Wohlfahrts-, Bildungs-, Wohnungs- und Gesundheitspolitik, die alle eine Vergrößerung städtischer Ungleichheit bewirkt. Diese Dy- namik lässt sich mit der folgenden algebraischen Formel zusammenfassen: $(E>K) \times S$. Das bedeutet, dass sozioräumliche Abschließung durch eine Kombination determiniert ist, in der Klasse von Ethnizität übertrumpft und durch den Staat verstärkt wird. Im Gegensatz dazu geht in Frankreich/Westeuropa die Klasse der Ethnizität bei der Verbannung voraus. Ferner wird die Marginalisierung vom Staat stark abgefedert und durch eine Kombination aus universalistischem Sozialschutz und zielgerichteter Interventionen gemildert, deren Zweck in einer Kontrolle städtischer Devolution liegt. Jene Erkenntnis liefert uns die folgende Formel: $(K>E) / S$.

Diese Ausführungen erklären das erste Wort im Titel des vorliegenden Beitrags: „Gestaltung städtischer AbschlieBung im 21. Jahrhundert.“ Es zeigt sich, dass der Staat auf beiden Seiten des Atlantiks der große Gestalter urbaner Marginalität ist, durch Unterlassung oder durch Auftragszahlung. Der Staat ist die Kraft, die durch die durchgeführte Verteilung von Menschen, Ressourcen und Aktivitäten die Parameter entlang des Raumes setzt, wie er vom Diagramm der sozioräumlichen Abschließung beschrieben wird. Durch seine vielfältigen Programme, angefangen bei der Stadtplanung, über ökonomische (De)Regulierung, Fiskalpolitik und Infrastrukturinvestitionen, bis hin zur räumlich differenzierten Bereitstellung öffentlicher Kerngüter wie Wohnungsbau, Bildung, Gesundheit, Wohlfahrt und Innere Sicherheit, bestimmt der Staat das Ausmaß der Distanz zwischen der Spitze und dem Boden der städtischen Ordnung; er bestimmt die Fahrzeuge, Pfade und die Bequemlichkeit, mit denen die Distanz zurückgelegt werden mag, und auch jene Formen sozioräumlicher Abschließung, die sich etablieren und auch wachsen (ob entmachtete und diffamierte Kategorien in ein Ghetto, in ein ethnisches Cluster oder in einen Slum eingeschlossen werden; wie groß das Gefängnissystem ist; wie abgeschlossen und isoliert Oberschichtviertel sind etc.). Durch seine Struktur und seine Politiken, durch seine schematisierten Aktionen und Trägheiten bestimmt der Leviathan die Entfaltungsmöglichkeiten, die Ausbreitung und die Intensität der Marginalität in der Stadt. Dies impliziert, dass StadtplanerInnen und ArchitektInnen, sofern sie bei der Formung der gebildeten Umwelt mitwirken, an der Raumproduktion der sozioräumlichen Verbannung teilhaben. Und sie werden verstärkt in die Gestaltung städtischer Abschließung involviert sein, da entwickelte Gesellschaften zunehmend auf räumliche „Lösungen“ zur Behandlung schwelender sozialer Probleme in der dualisierenden Metropole vertrauen.

Acknowledgements. Ich möchte Herrn Benedikt Kuhnen sehr herzlich für die Übersetzung dieses Beitrages aus dem amerikanischen Englisch danken. Auch gilt den Gutachtern dieses Artikels mein Dank für die konstruktiven Kommentare und Hinweise.

Edited by: O. Söderström

Reviewed by: two anonymous referees 


\section{Literatur}

Agier, M.: L'Invention de la ville, Banlieues, townships, invasions et favelas, Paris, 1999.

Agier, M.: Gérer les indésirables. Des camps de réfugiés au gouvernement humanitaire, Paris, 2008.

Atkinson, B. und Blandy, S. (Hrsg.): Gated Communities: International Perspectives, New York, 2006.

Blakely, E. und Snyder, M.: Fortress America: Gated Communities in the United States, Washington, DC, 1999.

Bourdieu, P.: The Forms of Capital, in: Handbook of Theory and Research for the Sociology of Education, Herausgeber: Richardson, J. G., New York, 241-258, 1986.

Calimani, R.: The Ghetto of Venice: A History, New York, 1987.

Cornell, S.: The Return of the Native: American Indian Political Resurgence, Oxford, 1988.

Drake, St. Clair und Cayton, H.: Black Metropolis: A Study of Negro Life in a Northern City, Chicago, 1945/1962.

Evans, I. T.: Bureaucracy and Race: Native Administration in South Africa, Berkeley, 1997.

Gregory, J. N.: The Southern Diaspora: How the Great Migrations of Black and White Southerners Transformed America, Chapel Hill, 2007.

Hall, P.: Cities of Tomorrow: An Intellectual History of Urban Planning and Design in the Twentieth Century, Malden, MA, 1988.

Kolchin, P.: American Slavery : 1619-1877, New York, 1993.

Liauzu, C. (Hrsg.): Dictionnaire de la colonisation française, Paris, 2007.

Lieberson, S.: A Piece of the Pie: Blacks and White Immigrants Since 1880, Berkeley, 1980.

Musterd, S., Murie, A., und Kesteloot, C. (Hrsg.): Neighbourhoods of Poverty: Urban Social Exclusion and Integration in Comparison, London, 2006.

Murray, M. J.: Taming the Disorderly City: The Spatial Landscape of Johannesburg After Apartheid, Ithaca, 2008.

Osofsky, G.: Harlem: The Making of a Ghetto - Negro New York, 1890-1930, New York, 1971.

The Oxford English Dictionary, second Edition, edited by: Simpson, J. and Weiner, E., Oxford University Press, Oxford, 1989.

Pinçon, M. und Pinçon-Charlot, M.: Les Ghettos du Gotha. Comment la bourgeoisie défend ses espaces, Paris, 2007.

Phillips, K. L.: Alabama North: African-American Migrants, Community, and Working-Class Activism in Cleveland, 1915-1945, Urbana, 1999.

Philpott, T. L.: The Slum and the Ghetto: Immigrants, Blacks, and Reformers in Chicago, 1880-1930, New York, 1978.
Sennett, R.: Flesh and Stone: The Body and the City in Western Civilization, New York, 1994.

Spear, A. H.: Black Chicago: The Making of a Negro Ghetto, 18901920, Chicago, 1968.

Stow, K. R.: Alienated Minority: The Jews of Medieval Europe, Cambridge, 1992.

Stébé, J.-M.: La Crise des banlieues. Sociologie des quartiers sensibles, Paris, 2007.

Trotter Jr., J. W. (Hrsg.): The Great Migration in Historical Perspective: New Dimensions of Race, Class, and Gender, Bloomington, 1991.

Wacquant, L.: Deadly Symbiosis: When Ghetto and Prison Meet and Mesh, Punishment \& Society 3, S. 95-133 (Deutsche Fassung: "Tödliche Symbiose. Wenn Ghetto und Gefängnis sich verbinden.“, in: Theorie als Kampf? Zur politischen Soziologie Pierre Bourdieus, Herausgeber: Bittlingmayer, U. H., Kastner, J., und Rademacher, C., Opladen, 265-313, 2001.

Wacquant, L.: "Ghetto.“, Herausgeber: Smelser, N. J. und Baltes, P. B., in: International Encyclopedia of the Social and Behavioral Sciences. London (Deutsche Fassung: "Was ist ein Ghetto? Konstruktion eines soziologischen Konzepts", in: Prokla: Zeitschrift für kritische Sozialwissenschaft (Berlin), 34-1, März, 133-148), 2004.

Wacquant, L.: Das Janusgesichte des Ghettos und andere Essays, Basel, Berlin, 2006.

Wacquant, L.: Territorial Stigmatization in the Age of Advanced Marginality, in: Thesis Eleven, 91 (November), 66-77 (Deutsche Fassung: "Territoriale Stigmatisierung im Zeitalter fortgeschrittener Marginalität.“, in: Das Argument (Berlin), 271, Juni 2007, 399-409), 2007.

Wacquant, L.: Urban Outcasts: A Comparative Sociology of Advanced Marginality (Deutsche Fassung: Die Verdammten dieser Stadt, Berlin, Springer, 2014), Cambridge, 2008.

Wacquant, L.: Punishing the Poor: The Neoliberal Government of Social Insecurity. Durham; London. (Deutsche Fassung: Bestrafen der Armen. Zur neoliberalen Regierung der sozialen Unsicherheit, Leverkusen, 2009), 2009a.

Wacquant, L.: The Body, the Ghetto and the Penal State, Qual. Sociol., 32, 101-129, 2009b.

Weber, M.: The City, Glencoe, 1921/1958.

Wilson, W. J.: The Truly Disadvantaged: The Inner City, the Underclass, and Public Policy, Chicago, 1987.

Wirth, L.: The Ghetto, Chicago, 1928.

Wirth, L.: Urbanism as a Way of Life, American Journal of Sociology, 4, 1-24 (reprinted in Wirth, L. (1964): On Cities and Social Life, Chicago, 60-83), 1938. 